\title{
Fatores associados à síndrome metabólica em idosos residentes em comunidade
}

\author{
Factors associated with metabolic syndrome in elderly residents in community \\ Los factores associados a la síndrome metabólico en ancianos residentes del comunidade
}

Recebido: 07/08/2021 | Revisado: 16/08/2021 | Aceito: 06/10/2021 | Publicado: 10/10/2021

Tuany Santos Souza

ORCID: https://orcid.org/0000-0003-0165-4201 Universidade Estadual do Sudoeste da Bahia, Brasil E-mail: tuanysouza.s@uesb.edu.br

José Ailton de Oliveira Carneiro

ORCID: https://orcid.org/0000-0001-5095-0301

Universidade Estadual do Sudoeste da Bahia, Brasil

E-mail: hitoef@yahoo.com.br

Silvania Moraes Costa

ORCID: https://orcid.org/0000-0002-6607-6177

Universidade Estadual do Sudoeste da Bahia, Brasil

E-mail: silvaniamoraescosta@hotmail.com

Yndiara Novaes Santos Oliveira

ORCID: https://orcid.org/0000-0001-7222-938X

Universidade Estadual do Sudoeste da Bahia, Brasil E-mail: yndiara@msn.com

Djanilson Barbosa dos Santos

ORCID: http://orcid.org/0000-0002-6128-1155 Universidade Federal do Recôncavo da Bahia, Brasil E-mail: djanilson@ufrb.edu.br

Cézar Augusto Casotti

ORCID: https://orcid.org/0000-0001-6636-8009 Universidade Estadual do Sudoeste da Bahia, Brasil E-mail: cacasotti@uesb.edu.br

\begin{abstract}
Resumo
Objetivo: verificar a prevalência e fatores associados à síndrome metabólica (SM) em idosos residentes em comunidade. Metodologia: trata-se de um estudo transversal, aninhado a uma coorte, de base populacional, com 259 idosos de idade $\geq 60$ anos do município de Aiquara-BA. A coleta de dados foi realizada de janeiro a junho de 2015, em três fases: aplicação de questionário no domicílio; mensuração de medidas antropométricas; realização de exames laboratoriais. A SM foi identificada utilizando-se os critérios definidos pelo National Cholesterol Education Program's - Adult Treatment Panel III. Realizou-se estatística descritiva e inferencial. A associação entre a SM e as variáveis independentes foram verificadas por meio de análises brutas e ajustadas usando a regressão de Poisson, com cálculo robusto de razões de prevalência (RP), intervalo de confiança de $95 \%$ (IC95\%), com p-valor $<0,05$, adotando um modelo de análise hierarquizada. Resultados: a prevalência de SM entre os idosos foi de 45,6\% e os principais fatores que permaneceram associados após análise ajustada foi o sexo feminino (RP=1,65; IC95\% 1,25-2,18) e o IMC, sendo o sobrepeso um fator de risco ( $\mathrm{RP}=1,67$; IC95\% 1,28-2,17) e o baixo peso um fator de proteção $(\mathrm{RP}=0,24$; IC95\% 0,10-0,56). Conclusão: conclui-se que a prevalência de SM nos idosos residentes em Aiquara-BA é alta e está associada ao sexo feminino e à obesidade.
\end{abstract}

Palavras-chave: Síndrome x metabólica; Idosos; Doenças cardiovasculares.

\begin{abstract}
Objective: prevalence, prevalence and factors associated with metabolic syndrome (MS) in community-dwelling elderly. Methodology: this is a cross-sectional study, nested in a population-based cohort, with 259 elderly people aged $\geq 60$ years from the city of Aiquara-BA. Data collection was carried out from January to June 2015, in three phases: questionnaire request at home; measurement of anthropometric measurements; carrying out laboratory tests. The MS was identified using the criteria defined by the National Cholesterol Education Program's - Adult Treatment Panel III. Descriptive and inferential statistics were performed. The association between MS and independent variables were verified through crude and adjusted analyzes using Poisson regression, with robust calculation of prevalence prevalence $(\mathrm{PR}), 95 \%$ confidence interval $(95 \% \mathrm{CI})$, with $\mathrm{p}$-value $<0.05$, adopting a hierarchical analysis model. Results: the prevalence of MS among the elderly was $45.6 \%$ and the main factors that remained associated after the adjusted analysis were female gender ( $\mathrm{PR}=1.65 ; 95 \% \mathrm{CI} 1.25-2.18)$ and BMI, being overweight a risk factor $(\mathrm{PR}=1.67 ; 95 \% \mathrm{CI} 1.28-2.17)$ and underweight a protection factor $(\mathrm{PR}=0.24 ; 95 \% \mathrm{CI} 0.10-0.56)$. Conclusion: it's
\end{abstract}


concluded that the prevalence of MS in the elderly living in Aiquara-BA is high and is associated with female gender and obesity.

Keywords: Metabolic syndrome X; Elderly; Cardiovascular diseases.

\begin{abstract}
Resumen
Objetivo: prevalencia, prevalencia y factores asociados al síndrome metabólico (SM) en ancianos residentes en la comunidad. Metodología: se trata de un estudio transversal, anidado en una cohorte poblacional, con 259 ancianos $\geq$ 60 años de la ciudad de Aiquara-BA. La recogida de datos se realizó de enero a junio de 2015, en tres fases: solicitud de cuestionario en domicilio; medición de medidas antropométricas; Realización de pruebas de laboratorio. El SM se identificó utilizando los criterios definidos por el Panel III de Tratamiento de Adultos del Programa Nacional de Educación sobre el Colesterol. Se realizó estadística descriptiva e inferencial. La asociación entre SM y variables independientes se verificó mediante análisis brutos y ajustados mediante regresión de Poisson, con cálculo robusto de prevalencia prevalencia (RP), intervalo de confianza del 95\% (IC 95\%), con p -valor <0,05, adoptando un criterio jerárquico modelo de análisis. Resultados: la prevalencia de SM entre los ancianos fue del 45,6\% y los principales factores que permanecieron asociados tras el análisis ajustado fueron el sexo femenino $(\mathrm{RP}=1,65$; IC 95\% 1,25-2,18) y el IMC, siendo el sobrepeso un factor de riesgo $(\mathrm{RP}=1,67 ; 95 \%$ CI 1,28-2,17) y bajo peso un factor de protección $(\mathrm{RP}=0,24 ; 95 \%$ CI 0,10-0,56). Conclusión: se concluye que la prevalencia del SM en los ancianos residentes en Aiquara-BA es alta y se asocia con el género femenino y la obesidad.
\end{abstract}

Palabras clave: Síndrome x metabólico; Ancianos; Enfermedades cardiovasculares.

\title{
1. Introdução
}

O aumento da população idosa é um fenômeno mundial e, no Brasil, vem acontecendo de forma acelerada devido à velocidade do processo de transição demográfica e epidemiológica evidenciado nas últimas décadas. Com isto observa-se o aumento da ocorrência de doenças crônicas não-transmissíveis - DCNT, incapacidades funcionais e uma crescente demanda do uso de serviços e tecnologias de saúde por essa população (Veras \& Oliveira, 2018). Neste contexto, dispor de adequada assistência, prevenção e promoção da saúde para a melhoria da qualidade de vida dos idosos torna-se um desafio, haja vista que esse grupo de indivíduos apresenta maior vulnerabilidade ao aparecimento de complicações que aumentam o risco de morbidade e mortalidade cardiovascular, sendo estes, muitas vezes, acarretados por doenças crônicas, como a síndrome metabólica - SM (Silva et al., 2012; Gragnolati et al., 2011)

De acordo com a I Diretriz Brasileira de Diagnóstico e Tratamento da Síndrome Metabólica - I-DBSM (Brasil, 2005), que se baseia nos critérios definidos pelo National Cholesterol Education Program's - Adult Treatment Panel III (NCEP ATP III, 2001), os principais componentes da SM são obesidade abdominal, hipertensão arterial sistêmica (HAS), dislipidemia (aumento dos níveis de triglicérides e diminuição dos níveis do HDL-colesterol) e distúrbio da glicemia (anormalidade da glicemia de jejum, tolerância diminuída à glicose ou presença de diabetes melitus). Assim, a alteração de três destes cinco parâmetros clínicos em um único indivíduo já se caracteriza como presença da SM (Brasil, 2005).

Ao longo do tempo, estudos epidemiológicos têm apontado associações da SM com o risco de desenvolvimento de diabetes melitus (DM), doenças cardiovasculares (DCV) e morte precoce (Dominguez \& Barbagallo, 2007; Nichols \& Moler, 2011; Tavares et al., 2018), além de maior utilização de serviços de saúde e custos médicos associados à prática da polifarmácia, o que acarreta no aparecimento de eventos adversos, devido, principalmente, à tendência da maior prevalência dos componentes isolados da síndrome entre os idosos (Souza et al., 2021), especialmente naqueles que vivem sob condições de sedentarismo (Coelho et al., 2021).

Vale ressaltar que os variados consensos para o critério de SM resultam em distintas estimativas de sua prevalência. Em estudos populacionais, estima-se que em regiões do Brasil, ela estaria entre 30,9 a 53,4\% (Rigo et al., 2009; Paula et al., 2010), sendo mais evidente a sua ocorrência com a elevação da faixa etária (Leitão \& Martins, 2012). Assim, diante do impacto da SM na saúde da população idosa, considerando este agravo como um importante fator de risco para DCV, DM e diversas complicações à saúde dos mesmos, objetivou-se com este estudo identificar a prevalência e fatores associados da 
síndrome metabólica em idosos residentes em comunidade.

\section{Metodologia}

\section{Delineamento e população do estudo}

Trata-se de um estudo epidemiológico transversal analítico, aninhado a uma coorte, de base populacional, realizado com idosos residentes em comunidade. O campo de estudo foi o município de Aiquara-BA, onde participaram idosos de ambos os sexos que atenderam aos seguintes critérios de elegibilidade: ter 60 anos ou mais de idade, não institucionalizados; com residência fixa na zona urbana; que não apresentaram déficit cognitivo de acordo com a avaliação do Mini Exame do Estado Mental (MEEM) (Folstein, Folstein \& Mchugh, 1975) e que aceitaram participar dos estágios da pesquisa, mediante assinatura do Termo de Consentimento Livre e Esclarecido (TCLE). Inicialmente foi realizado um censo da população, onde foram identificados 379 idosos no município de Aiquara-BA. Entretanto, 29 idosos foram excluídos do estudo por não terem condições de responder ao questionário e não possuírem acompanhante que os auxiliassem nas respostas e 7 por residirem na zona rural; 34 perdas por viagem, internamento hospitalar ou não localização após três tentativas em turnos diferentes; 20 recusas; 30 idosos não realizaram a coleta sanguínea. Assim, compuseram a amostra deste estudo os 259 idosos.

\section{Procedimentos de coleta de dados}

Os dados foram coletados em três fases: I. Aplicação de questionário padronizado no domicílio, realizado em janeiro de 2015, construído por meio da compilação de questionários SABE - Saúde, Bem-estar e Envelhecimento (Lebrão \& Duarte, 2003); BOAS - Brazil Old Age Schedule (Veras \& Dutra, 2008); IPAQ - Questionário Internacional de Atividade Física, versão adaptada para idosos (Mazo \& Benedetti, 2010), onde obteve-se informações sociodemográficas, comportamentais e de condições de saúde; II. Realização de medidas antropométricas em março de 2015, por fisioterapeutas treinados, com dias e horários previamente agendados com os idosos; III. Coleta de amostra sanguínea, para análises laboratoriais, realizada entre maio e julho de 2015, na Secretaria Municipal de Saúde e no domicílio para os idosos acamados, sob a recomendação de um jejum prévio de 12h. As análises foram realizadas no Laboratório de Saúde Pública do Centro de Referência em doenças endêmicas Pirajá da Silva, no município de Jequié-BA.

\section{Determinações bioquímicas}

A avaliação bioquímica foi realizada por meio de tecnologia automatizada SELLECTRA II ${ }^{\circledR}$, onde foram determinados os níveis séricos da glicemia de jejum e triglicérides pelo método enzimático colorimétrico, e o nível de colesterol HDL através de precipitação direta.

\section{Determinação da pressão arterial}

A pressão arterial foi aferida com uso de aparelho semiautomático digital da marca HEM 742 (Omron®), de acordo com procedimentos padronizados pela VII Diretriz Brasileira de Hipertensão Arterial (Malachias et al., 2016). Foram realizadas duas aferições, com intervalo de cinco minutos entre as medidas. Os níveis pressóricos foram considerados alterados quando a média das duas aferições da pressão arterial foi $\geq 130 / 80 \mathrm{mmHg}$ e/ou quando havia uso de medicamentos antihipertensivos.

\section{Determinação de medidas antropométricas}

A circunferência abdominal é a medida mais recomendada por ser o índice antropométrico mais representativo da gordura intra-abdominal e de aferição mais simples e reprodutível (Brasil, 2005). A medida foi aferida em triplicata, na altura 
da cicatriz umbilical, no meio da distância entre a crista ilíaca e o rebordo costal inferior, com o auxílio de uma fita métrica flexível não elástica com trava, com precisão de $1 \mathrm{~mm}$ e validada em território nacional (Sanny®). Foi solicitando ao idoso que ficasse de pé, com a menor quantidade de roupas possível, para facilitar a mensuração das medidas. A leitura foi realizada na direção do olhar do avaliador, com a fita perpendicular ao solo, de forma firme na região a ser avaliada, mas sem comprimir a pele do avaliado.

\section{Variável dependente}

A SM foi identificada de acordo com os parâmetros definidos pela I Diretriz Brasileira de Diagnóstico e Tratamento da Síndrome Metabólica (Brasil, 2005), que se baseia nos critérios definidos pelo National Cholesterol Education Program's Adult Treatment Panel III (NCEP - ATP III, 2001). Assim, considerou-se presença de SM quando o indivíduo apresentou pelo menos três dos seguintes componentes, segundo o NCEP-ATP III: pressão arterial sistólica $\geq 130$ ou pressão arterial diastólica $\geq 85 \mathrm{mmHg}$, ou uso de medicamento anti-hipertensivo; glicemia de jejum $\geq 110 \mathrm{mg} / \mathrm{dL}$ ou uso de hipoglicemiantes; triglicerídeos $\geq 150 \mathrm{mg} / \mathrm{dL}$; colesterol $\mathrm{HDL}<40 \mathrm{mg} / \mathrm{dL}$ para os homens e $<50 \mathrm{mg} / \mathrm{dL}$ para as mulheres e circunferência abdominal $\geq 88 \mathrm{~cm}$ para as mulheres $\geq 102 \mathrm{~cm}$ para os homens.

\section{Variáveis independentes}

1. Variáveis sociodemográficas: sexo; faixa etária em anos completos $(60-69,70-79$ e $\geq 80)$; cor da pele segundo o entrevistador (negro e não-negro); escolaridade em anos de estudo ( $\leq 8$ anos e $>8$ anos); arranjo familiar (com companheiro e sem companheiro) renda ( $\leq 1$ salário mínimo; > 1 salário mínimo);

2. Variáveis comportamentais: uso abusivo de álcool (sim e não); tabagismo (sim e não); nível de atividades físicas, obtido a partir do Questionário Internacional de Atividade Física - IPAQ ( $\geq 150$ minutos: suficientemente ativo; $<150$ minutos: insuficientemente ativo) (Mazo \& Benedetti, 2010); uso dos serviços de saúde por ano ( $\geq 2$ vezes/ano; 1 vez/ano; nenhuma);

3. Variáveis laboratoriais e de condições de saúde: determinação das concentrações séricas de colesterol total (VR: $<200 \mathrm{mg} / \mathrm{dL}$ ); colesterol LDL (VR: <130 mg/dL); vitamina D (Suficiente: >30 pg/dL; Insuficiente: 20-30 pg/dL; Deficiente: $<20$ pg/dL); Hormônio Tireoestimulante - TSH (VR: 0,4 a 4,5 $\mu \mathrm{UI} / \mathrm{ml}$ ); Índice de massa corpórea - IMC (Baixo Peso: $\leq 22$ $\mathrm{kg} / \mathrm{m}^{2}$; Eutrófico: $>22$ e $<27 \mathrm{~kg} / \mathrm{m}^{2}$; Sobrepeso: $\geq 27 \mathrm{~kg} / \mathrm{m}^{2}$ ); uso de medicamentos para algum dos componentes da SM (sim e não); comorbidades (nenhuma; 2 ou mais).

\section{Análise estatística}

As análises descritivas incluíram cálculos de frequências absoluta e relativa para as variáveis categóricas e médias e desvio-padrão para as variáveis contínuas. A prevalência de SM foi calculada, levando-se em consideração a população estudada e o total de expostos. A associação entre SM e as variáveis independentes foram verificadas por meio de análises brutas e ajustadas usando a regressão de Poisson, com cálculo robusto de razões de prevalência (RP) e intervalo de confiança de 95\% (IC95\%). Na análise bruta a prevalência de SM foi calculada para cada categoria das variáveis independentes e a significância estatística obtida por meio do teste de Wald de heterogeneidade. As variáveis que apresentaram significância estatística de pelo menos $20 \%(\mathrm{p} \leq 0,20)$ nas análises brutas permaneceram na análise ajustada, com base na ordem de um modelo hierárquico para a determinação dos resultados, em que as variáveis foram incluídas na ordem de um modelo conceitual multinível onde inicialmente foram incluidas as variáveis sociodemográficas no nível 1, seguidas das variáveis comportamentais no nível 2 e, por fim, foram incluídas as variáveis laboratoriais e de condições de saúde no nível 3 (Figura 1). O efeito de cada variável independente sobre o resultado foi controlado pelas variáveis do mesmo nível e de níveis mais 
elevados no modelo. O nível de significância adotado no estudo foi de $5 \%$. Os dados foram tabulados no softwere Excel ${ }^{\circledR} \mathrm{e}$ analisados no programa Statistical Package for Social Sciences para Windows (SPSS ${ }^{\circledR}$ 21.0, 2013, SPSS, Inc, Chicago, IL).

\section{Aspectos éticos}

O presente estudo está vinculado ao projeto de pesquisa "Condições de Saúde e Estilo de Vida de idosos residentes em município de pequeno porte". Foi submetido e aprovado pelo Comitê de Ética em Pesquisa da Universidade Estadual do Sudoeste da Bahia (CEP-UESB) sob número do Parecer: 1.575.825 e atende ao disposto na Resolução 466/2012 do Conselho Nacional de Saúde (Brasil, 2012).

Figura 1. Modelo hierárquico de análise.

\begin{tabular}{|c|}
\hline Nivel 1 - Variáveis sociodemograficas \\
Sexo \\
Faixa Etária \\
Cor da Pele \\
Escolaridade \\
Arranjo Familiar \\
Renda \\
Nivel 2- Variáveis comportamentais \\
Consumo de Álcool \\
Tabagismo \\
Nivel de atividades fisicas \\
Uso dos serviços de saúde
\end{tabular}

\begin{tabular}{|c|}
\hline $\begin{array}{l}\text { Nivel3-Vantiaveis latoratoriais e de } \\
\text { condições de saúde }\end{array}$ \\
\hline Colesterol Total \\
\hline Colesterol LDL \\
\hline TSH \\
\hline T4 livre \\
\hline Vitamina D \\
\hline Índice de Massa Corpórea (IMC) \\
\hline $\begin{array}{l}\text { Uso de medicamentos para componentes } \\
\text { da SM }\end{array}$ \\
\hline Comorbidades \\
\hline
\end{tabular}

\section{Sindrome Metabólica}

Fonte: Autores. 


\section{Resultados}

Participaram do estudo 259 idosos, com faixa etária entre 60 e 95 anos, média de idade de 71,8 ( $\pm 7,8)$, sendo destes $56,8 \%$ do sexo feminino. Prevaleceram idosos com faixa etária entre 70-79 anos (42,1\%), negros (71,7\%), com baixa escolaridade $(78,8 \%)$ e de baixa renda $(85,4 \%)$. As demais características sociodemográficas, comportamentais, laboratoriais e de condições de saúde da população estão apresentadas na Tabela 1. A prevalência de SM na população estudada foi de 45,6\%. De acordo com os critérios do NCEP/ATP III, 51,3\% dos participantes apresentavam pelo menos três dos cinco componentes da SM, 35\% quatro componentes e 13,7\% apresentavam todos os componentes. Dentre eles, o mais prevalente foi a HAS $(89,0 \%)$, seguido de obesidade abdominal (82,2\%), dislipidema (65,3\%), hipertrigliceridemia (61,0\%) e hiperglicemia $(50,8 \%)$.

Tabela 1. Características da população de estudo. Aiquara-BA, Brasil.

\begin{tabular}{|c|c|c|c|}
\hline Variáveis & \% resposta & $\mathbf{N}$ & $\%$ \\
\hline Sexo & 100 & & \\
\hline Masculino & & 112 & 43,2 \\
\hline Feminino & & 147 & 56,8 \\
\hline Faixa etária & 100 & & \\
\hline $60-69$ & & 102 & 39,4 \\
\hline $70-79$ & & 109 & 42,1 \\
\hline$\geq 80$ & & 48 & 18,5 \\
\hline Cor da pele & 99,6 & & \\
\hline Negro & & 185 & 71,7 \\
\hline Não Negro & & 73 & 28,3 \\
\hline Escolaridade (anos de estudo) & 97,3 & & \\
\hline$<8$ anos & & 193 & 78,8 \\
\hline$>8$ anos & & 52 & 21,2 \\
\hline Arranjo Familiar & 98,8 & & \\
\hline Com companheiro (a) & & 126 & 49,2 \\
\hline Sem companheiro (a) & & 130 & 50,8 \\
\hline Renda* & 98,1 & & \\
\hline > 1 salário mínimo & & 37 & 14,6 \\
\hline$\leq 1$ salário mínimo & & 217 & 85,4 \\
\hline Consumo de álcool & 98,8 & & \\
\hline Não & & 201 & 78,5 \\
\hline Sim & & 55 & 21,5 \\
\hline Tabagismo & 92,3 & & \\
\hline Não & & 214 & 89,5 \\
\hline Sim & & 25 & 10,5 \\
\hline Nível de atividades físicas & 100 & & \\
\hline Insuficientemente ativo & & 121 & 46,7 \\
\hline Suficientemente ativo & & 138 & 53,3 \\
\hline Uso de serviços de saúde & 95,8 & & \\
\hline$\geq 2$ vezes/ano & & 200 & 80,6 \\
\hline 1 vez/ano & & 21 & 8,5 \\
\hline Nenhuma & & 27 & 10,9 \\
\hline Colesterol total & 99,2 & & \\
\hline Normal & & 111 & 43,2 \\
\hline Alterado & & 146 & 56,8 \\
\hline Colesterol LDL & 95,3 & & \\
\hline Normal & & 127 & 52,5 \\
\hline Alterado & & 115 & 47,5 \\
\hline TSH & 93,1 & & \\
\hline Normal & & 229 & 95,0 \\
\hline Alterado & & 12 & 5,0 \\
\hline T4 livre & 96,5 & & \\
\hline Normal & & 248 & 99,2 \\
\hline Alterado & & 2 & 0,8 \\
\hline Vitamina D & 88,4 & & \\
\hline Suficiente & & 97 & 42,4 \\
\hline Insuficiente & & 93 & 40,6 \\
\hline Deficiente & & 39 & 17,0 \\
\hline
\end{tabular}




\begin{tabular}{|c|c|c|c|}
\hline IMC & 86,1 & & \\
\hline Eutrófico & & 80 & 35,9 \\
\hline Baixo peso & & 48 & 21,5 \\
\hline Sobrepeso & & 95 & 42,6 \\
\hline Uso de medicamentos ${ }^{* *}$ & 100 & & \\
\hline Não & & 162 & 62,8 \\
\hline Sim & & 96 & 37,2 \\
\hline Síndrome metabólica & 100 & & \\
\hline Não & & 141 & 54,4 \\
\hline Sim & & 118 & 45,6 \\
\hline
\end{tabular}

*Salário mínimo 2015 = R \$ 788,00; **Uso de medicamentos para algum dos componentes da síndrome metabólica; IMC: Índice de Massa Corpórea. Fonte: Autores.

A Tabela 2 apresenta a prevalência da SM, de acordo com as variáveis independentes do estudo, onde as variáveis sociodemográficas, comportamentais, laboratoriais e de condições de saúde que atingiram significância estatística $(p \leq 0,20)$ foram selecionadas para serem incluídas na análise ajustada. Assim, na análise bruta a SM foi significativamente mais frequente nos idosos do sexo feminino, não negros, que vivem sem companheiro, que fazem uso de bebida alcóolica e tabaco, que não frequentaram o serviço de saúde nenhuma vez ao ano, com deficiência de vitamina D, com IMC alterado (sobrepeso e baixo peso) e que faz uso de medicamentos para algum dos critérios da síndrome. Após os ajustes intra e interníveis de acordo com o modelo hierárquico, as variáveis sociodemográficas (cor da pele e arranjo familiar), comportamentais (tabagismo e uso dos serviços de saúde), laboratoriais e de condições de saúde (vitamina $\mathrm{D}$, uso de medicamentos e comorbidades) não permaneceram no modelo final, por não terem encontrado critério de significância $(\mathrm{p} \leq 0.20)$.

Tabela 2. Prevalência de síndrome metabólica e sua relação com as variáveis independentes do estudo. Aiquara-BA, Brasil.

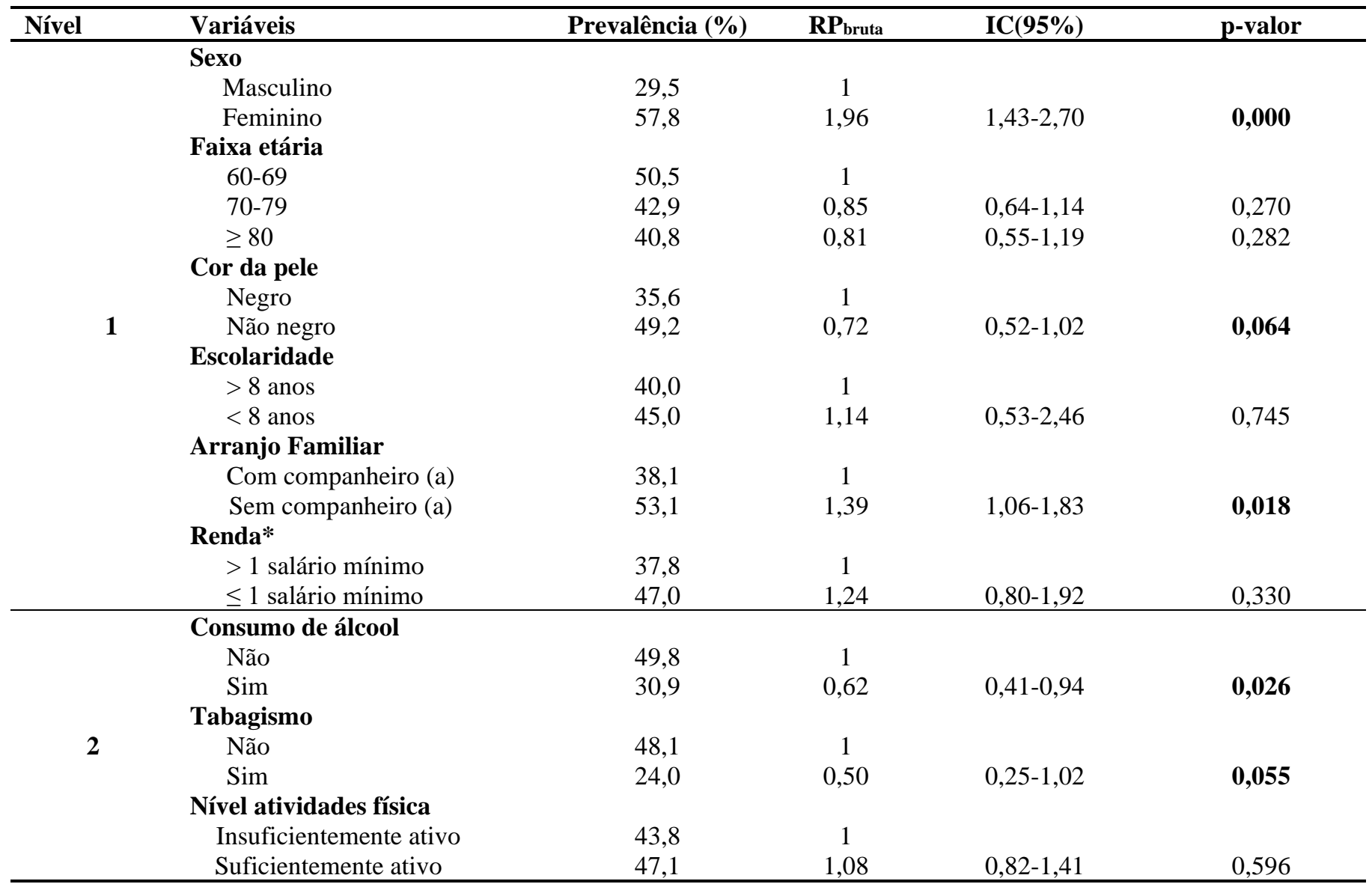




\begin{tabular}{|c|c|c|c|c|c|}
\hline & \multicolumn{5}{|l|}{ Uso serviços de saúde } \\
\hline & $\geq 2$ vezes/ano & 48,0 & 1 & & \\
\hline & $1 \mathrm{vez} / \mathrm{ano}$ & 38,1 & 0,79 & $0,45-1,40$ & 0,421 \\
\hline & Nenhuma & 29,6 & 0,62 & $0,34-1,12$ & 0,110 \\
\hline & \multicolumn{5}{|l|}{ Colesterol total } \\
\hline & Normal & 45,0 & 1 & & \\
\hline & Alterado & 45,9 & 1,02 & $0,75-1,31$ & 0,993 \\
\hline & \multicolumn{5}{|l|}{ Colesterol LDL } \\
\hline & Normal & 42,5 & 1 & & \\
\hline & Alterado & 47,0 & 1,11 & $0,83-1,46$ & 0,488 \\
\hline & \multicolumn{5}{|l|}{ TSH } \\
\hline & Normal & 45,9 & 1 & & \\
\hline & Alterado & 50,0 & 1,09 & $0,61-1,95$ & 0,771 \\
\hline & \multicolumn{5}{|l|}{ T4 livre } \\
\hline & Normal & 46,6 & 1 & & \\
\hline 3 & \multirow{2}{*}{\multicolumn{5}{|c|}{$\begin{array}{r}\text { Alterado } \\
\text { Vitamina D }\end{array}$}} \\
\hline & & & & & \\
\hline & Suficiente & 40,2 & 1 & & \\
\hline & Insuficiente & 49,5 & 1,23 & $0,89-1,69$ & 0,202 \\
\hline & Deficiente & 53,8 & 1,34 & $0,92-1,95$ & $\mathbf{0 , 1 3 0}$ \\
\hline & \multicolumn{5}{|l|}{ IMC } \\
\hline & Eutrófico & 42,5 & 1 & & \\
\hline & Baixo peso & 10,4 & 0,25 & $0,10-0,58$ & 0,001 \\
\hline & Sobrepeso & 77,9 & 1,83 & $1,39-2,42$ & $\mathbf{0 , 0 0 0}$ \\
\hline & \multicolumn{5}{|l|}{ Uso de medicamentos** } \\
\hline & Não & 38,3 & 1 & & \\
\hline & Sim & 58,3 & 1,52 & $1,16-1,97$ & 0,001 \\
\hline & \multicolumn{5}{|l|}{ Comorbidades } \\
\hline & Nenhuma & 33,3 & 1 & & \\
\hline & 2 ou mais & 45,7 & 1,37 & $0,28-6,83$ & 0,700 \\
\hline
\end{tabular}

*Salário mínimo 2015 = R \$ 788,00 **Uso de medicamentos para algum dos componentes da síndrome metabólica; IMC: Índice de Massa Corpórea. Fonte: Autores.

A Tabela 3 apresenta o modelo final da regressão após análise ajustada, onde verifica-se que a SM esteve associada às variáveis sexo e IMC, sendo a razão de prevalência deste agravo maior em idosos do sexo feminino e com sobrepeso, e tendo o baixo peso um fator de proteção para a SM nesta população.

Tabela 3. Análise ajustada* da síndrome metabólica com as variáveis independentes do estudo. Aiquara-BA, Brasil.

\begin{tabular}{lccc}
\hline Variáveis & RPajustada & IC95\% & p-valor \\
\hline Sexo & 1 & & \\
$\quad$ Masculino & 1,65 & $1,25-2,18$ & $\mathbf{0 , 0 0 1}$ \\
$\quad$ Feminino & & & \\
Consumo de bebida alcóolica & 1 & & 0,103 \\
$\quad$ Não & 0,76 & $0,54-1,06$ & \\
$\quad$ Sim & & & \\
IMC & 1 & & $\mathbf{0 , 0 0 1}$ \\
$\quad$ Eutrófico & 0,24 & $0,10-0,56$ & $\mathbf{0 , 0 0 0}$ \\
$\quad$ Baixo Peso & 1,67 & $1,28-2,17$ & \\
$\quad$ Sobrepeso
\end{tabular}

\section{Discussão}

*Análise ajustada por sexo e IMC. Fonte: Autores.

A prevalência de SM nos idosos de Aiquara-BA foi de 45,6\%. Embora no Brasil ainda não haja estudos sobre a prevalência de SM com dados representativos da população geral, é descrito na literatura uma grande variação nas taxas de prevalência de SM em idosos, provavelmente por causa do perfil da população estudada e dos critérios utilizados para a 
discriminação da síndrome, tal como o estudo feito por Saad et al. (2014), que encontraram prevalência semelhante numa amostra de 243 idosos na cidade de Niterói-RJ (45,2\%). Outros autores descreveram percentuais maiores como Wachholz e Masuda (2009) em estudo com 190 idosos de Colombo-PR (51,6\%); Franco et al. (2009) com 120 indivíduos hipertensos, sendo 57 idosos da cidade de Cuiabá-MT (70,8\%) e Cloos et al. (2016) com 186 idosos de um Serviço de Geriatria em Porto Alegre-RS (58,6\%).

Entretanto, prevalências menores foram identificadas em Viçosa-MG em estudo conduzido com 113 idosas cadastradas no Programa Saúde da Família (30,9\%) (Paula et al., 2010) e em São Carlos-SP com 1116 indivíduos entre 30-79 anos, sendo a prevalência de SM 21,7\% entre idosos com 60-69 anos e 19,3\% entre 70-79 anos (Gronner et al., 2011). Ressalta-se que as diferenças entre as prevalências podem estar relacionadas ao tamanho da amostra, desenho do estudo, bem como a questões relacionadas com o estilo de vida, condições de saúde, diferenças regionais e perfil epidemiológico da população estudada.

Embora não haja valores da prevalência de SM representativos da população brasileira para comparação fidedigna a outros países, estudos internacionais também revelam prevalências variáveis da SM em idosos segundo o país em que residem. Na Pesquisa Nacional de Saúde e Nutrição (NHANES III) com 3423 adultos e idosos norte-americanos foi relatada uma prevalência de 51,5\% em indivíduos com idade $\geq 60$ anos (Ervin, 2009). Na Espanha foi descrita a prevalência de 46,8\% em 862 indivíduos com idade >65 anos (De Luis et al, 2010); na Finlândia encontraram 34,1\% em 539 idosos com idade > 70 anos (Saukkonen et al., 2012) e em análise transversal com 1535 idosos americanos de 70 a 89 anos, participantes do Estudo de Intervenções no Estilo de Vida e Independência para Anciãos (LIFE), encontrou-se prevalência de 49,8\% (Botoseneanu et al., 2015).

Vale ressaltar que a obesidade abdominal, hipertensão arterial sistêmica, dislipidemia e distúrbios da glicemia, principais componentes da SM, tendem a apresentar maior prevalência entre idosos (Souza et al., 2021). Em Aiquara-BA identificamos que 51,3\% dos idosos com SM apresentavam pelo menos três dos cinco componentes, $35 \%$ quatro componentes e 13,7\% todos os componentes. Dentre eles, o mais prevalente foi a HAS (89,0\%), fato também observado por outros autores (Closs, Feoli \& Schwanke, 2016; Vieira, Peixoto \& Silveira, 2014; Rosa et al., 2016). Entretanto, independente de qual componente esteja alterado, qualquer um deles eleva o risco de DCV e DM tipo 2. Portanto, o monitoramento é importante desde quando há apenas um componente alterado, pois o risco metabólico já existe e a abordagem clínica se faz necessária (Coelho et al., 2021).

$\mathrm{Na}$ análise ajustada da síndrome metabólica com as variáveis independentes deste estudo (sociodemográficas, comportamentais, laboratoriais e de condições de saúde) as únicas que permaneceram associadas à $\mathrm{SM}$, foram o sexo (RP= 1,65; IC95\% 1,25-2,18) e IMC alterado (sobrepeso RP=1,67; IC95\% 1,28-2,17) e (baixo peso RP=0,24; IC95\% 0,10-0,56).

No que diz respeito ao sexo, em Aiquara-BA a prevalência de SM foi significativamente maior nas idosas $(57,8 \%)$ que nos idosos (29,5\%). Esta associação também tem sido descrita por outros estudos, como observado em Coimbra-MG onde a prevalência nas mulheres idosas foi $40 \%$ e nos homens $22 \%$ (Paula et al., 2015), em Niterói-RJ em idosas atendidos em ambulatório foi 45,6\% e em idosos 44,4\% (Saad et al., 2014) e, em Virgem das Graças-MG em idosas 23,3\% e idosos 6,5\% (Pimenta, Gazzinelli \& Velásquez-Melendez, 2011). Alguns autores ressaltam que em populações com escassos recursos socioeconômicos, como a do presente estudo, as mulheres constituem-se no grupo mais vulnerável para a ocorrência de doenças crônicas não-transmissíveis por apresentarem maiores taxas de sedentarismo, obesidade e alterações metabólicas (Damião et al, 2007; Coelho et al., 2021). Variações na prevalência de SM de acordo com o sexo podem estar relacionadas a diferenças na distribuição de gordura corporal, tamanho e função dos adipócitos, prevalência de resistência insulínica, regulação hormonal do peso corporal e da adiposidade, além da influência do declínio de estrogênio durante a menopausa sobre os fatores de risco para SM (Pradhan, 2014). 
Vale salientar que o estrogênio é considerado como fator protetor para doenças cardiovasculares nas mulheres prémenopáusicas, e devido à redução deste hormônio em mulheres após a menopausa ocorre maior suscetibilidade ao aumento de gordura na região abdominal (Ferreira et al., 2008; Janssen et al., 2010). Em Aiquara-BA a maior prevalência da SM nas idosas ainda pode estar relacionada ao fato delas terem maiores proporções de obesidade abdominal quando comparadas aos homens (78,1\% vs 23,9\%), maiores níveis séricos de triglicerídeos (37,7\% vs 33,3\%), menores níveis séricos de HDL-c (48,6\% vs $25,9 \%$ ), bem como maior índice de massa corpórea (50,8\% vs 33,3\%). Um estudo realizado com mulheres nipo-brasileiras, com idade média de 60,8 anos com elevada prevalência de SM identificou melhoras significativas no perfil lipídico e antropométrico dessas pacientes, mediante dois anos de intervenções em hábitos alimentares e atividade física (Ferreira et al., 2008). Frente a este quadro, é consenso que a população idosa da cidade de Aiquara-BA, especialmente as mulheres, devem ser incentivadas a mudarem seu estilo de vida, quanto à adoção de hábitos alimentares mais saudáveis, associados à prática de atividades físicas mediante um acompanhamento multiprofissional efetivo (Mendes et al., 2012).

Ademais, neste estudo outro fator associado à SM foi o $\mathrm{IMC}$, apontando o sobrepeso $(\mathrm{RP}=1,67)$ como um fator de risco e o baixo peso como um fator de proteção $(\mathrm{RP}=0,24)$, o que reforça a relação fisiopatológica entre a adiposidade central e a resistência insulínica e distúrbios hormonais relacionados a vitamina D (Lima et al., 2017). Esse dado corrobora a outros estudos que também encontraram associação da SM com IMC aumentado (categoria sobrepeso) em idosos (Vieira, Peixoto \& Silveira, 2014; Pimenta, Gazzinelli \& Velásquez-Melendez, 2011). Esse resultado torna-se relevante ao se levar em consideração o grande impacto que a obesidade exerce tanto na morbidade quanto na mortalidade cardiovascular de idosos (Góis \& Veras, 2010). A associação entre SM e sobrepeso avaliado pelo IMC nesta população pode estar relacionada aos maus hábitos de saúde relacionados à alimentação e prática insuficiente de exercícios físicos, o que leva a uma inversão no perfil lipídico (baixo HDL-c e alto TG). Destarte, apesar de o IMC não ser uma medida específica para predizer a obesidade central, ele pode refletir o depósito ectópico de gordura no músculo esquelético, o que favoreceria a resistência à insulina, limitando posteriormente sua secreção (Botoseneanu et al., 2015), mecanismo esse envolvido na fisiopatogênese da SM.

Vale ressaltar que as questões relativas aos pontos de corte para avaliar IMC ainda são controversas e necessitam de mais estudos para validar os novos critérios desse indicador no diagnóstico de obesidade em idosos, visto que essa é uma medida de obesidade global, relacionada aos distúrbios metabólicos. Neste sentido, associações mais fortes e independentes são observadas com o aumento da circunferência abdominal, por se tratar de uma medida relacionada à obesidade central e que exerce maior influência sobre o risco cardiovascular (Fox et al., 2007), tal como é preconizado pela I-DBSM (Brasil, 2005). Assim, sugerimos que a combinação de IMC com medidas da distribuição de gordura pode ajudar a resolver alguns problemas do uso do IMC isolado, dessa forma verifica-se que tanto os efeitos da obesidade global quanto da obesidade centralizada são aditivos para a ocorrência da SM e devem ser cautelosamente monitoradas (Pimenta, Gazzinelli \& Velásquez-Melendez, 2011).

Este estudo apresentou algumas limitações como o fato de o desenho adotado nos impedir de identificar a temporalidade e causalidade das associações, bem como a ausência de alguns idosos em suas residências no momento das entrevistas, por trabalharem na zona rural ou por viagens, como também a falta de um acompanhante que auxiliassem os idosos com agravamento da cognição a responderem ao questionário, o que acarretou em algumas perdas.

Por outro lado, podem ser destacados alguns pontos que favorecem a validade deste estudo: sua característica censitária, a adoção de instrumentos de coleta validados e utilizados em vários países em estudos populacionais, aferição de medidas por técnicas apropriadas e realização de exames bioquímicos em Laboratório de Saúde Pública de referência. Cita-se ainda a validade dos ajustes das variáveis por meio de técnica de análise multivariada adequada para o tipo de delineamento do estudo. Destarte, ao considerar o delineamento transversal do presente estudo, é possível que a ocorrência de causalidade reversa tenha limitado também a análise das demais associações. Entretanto, alguns resultados apesar de estatisticamente não significativos, mostram-se relevantes e precisam ser melhor investigados, como maior prevalência de SM entre os idosos com 
baixa escolaridade, com outras comorbidades, com níveis séricos de colesterol total e LDL-c elevados e com alguns parâmetros hormonais alterados, como a vitamina D e TSH.

Por fim, é importante salientar que, em virtude da associação entre fatores modificáveis e a SM, medidas preventivas primárias devem ser adotadas para diminuir tanto a prevalência desse agravo, como de seus componentes isolados, com o intuito de minimizar os impactos na saúde dos idosos deste município. Entre essas ações, destaca-se o controle efetivo dos fatores de risco, principalmente da obesidade que, potencialmente, melhoraria o perfil lipídico e cardiometabólico dessa população. Propõe-se, ainda, que outros estudos, sobretudo longitudinais, sejam conduzidos nesta população para identificar os principais fatores que determinam essa condição de morbidade nesses idosos.

\section{Conclusão}

Conclui-se que idosos residentes em comunidade na zona urbana de Aiquara-BA, município de pequeno porte, com indicadores sociais desfavoráveis, apresentaram alta prevalência de SM e como fatores associados foram identificadas as variáveis sexo feminino e sobrepeso e como fator de proteção o baixo peso, avaliados através da medida do IMC. Considerando tais associações, torna-se relevante o desenvolvimento de estratégias de intervenção para diminuir a exposição destes idosos aos fatores de risco tanto da SM e de seus componentes isolados, quanto relacionados à obesidade, através de ações de vigilância epidemiológica que visem a promoção de hábitos de vida mais saudáveis e recuperação da saúde dos idosos em eminente risco cardiovascular.

\section{Agradecimentos}

À Coordenação de Aperfeiçoamento de Pessoal de Nível Superior e à Fundação de Amparo à Pesquisa do Estado da Bahia pelo financiamento do Projeto.

\section{Referências}

Botoseneanu, A., Ambrosius, W. T., Beavers, D. P., Rekeneire, N., Anton, S., Church, T. et al. (2015). Prevalence of metabolic syndrome and its association with physical capacity, disability, and self-rated health in Lifestyle Interventions and Independence for Elders Study participants. J Am Geriatr Soc, 63(2):22232 .

Brasil (2005). I Diretriz brasileira de diagnóstico e tratamento da síndrome metabólica. Arq. bras. cardiol., 84(supl. 1):3-28.

Brasil (2012). Resolução no . 466, de 12 de dezembro de 2012. Aprova diretrizes e normas regulamentadoras de pesquisas envolvendo seres humanos. Diário Oficial da União. 13 jun. 2013.

Closs, V. E., Feoli, A. M. P., \& Schwanke, C.H.A. (2016). Síndrome metabólica em idosos da atenção terciária em Porto Alegre, Rio Grande do Sul: associação com o Índice de Alimentação Saudável. Sci Med, 26(3):ID23422.

Coelho, J. M. F., Silva, A. S., Brito, E. X. dos S., Marques, E. M., Ponte, G. A., Gomes, Érica V. D., Lima, K. G., França, L. M. C., Lima, L. A. da S., Mêrces, M. C., Sampaio, L. M. de A., Fernandes, B. de S., Pinheiro, I. M., Galvão, L. R., \& Galvão, C. R. (2021). Sedentarism and Metabolic Syndrome in users of a Family Health Unit in Salvador- BA. Research, Society and Development, 10(1), e56910112195.

Damião, R., Pititto, B., Gimeno, S., \& Ferreira, S. (2007). Aspectos epidemiológicos e nutricionais da síndrome metabólica. In: Kac, G., Sichieri, R., Gigante, D.P. Epidemiologia nutricional. Rio de Janeiro: Editora Fiocruz/Editora Atheneu, 389-408.

Luis, D. A., Lopez Mongil, R., Gonzalez Sagrado, M., Lopez Trigo, J. A., Mora, P. F., Castrodeza Sanz, J.; Group Novomet (2010). Prevalence of metabolic syndrome with International Diabetes Federation criteria and ATP III program in patients 65 years of age or older. J Nutr Health Aging, 14(5):400-4.

Dominguez, L.J., \& Barbagallo, M. (2007). The cardiometabolic syndrome and sarcopenic obesity in older persons. J Cardiometab Syndr, 2 (3):183-9.

Ervin, R.B. (2009). Prevalence of metabolic syndrome among adults 20 years of age and over, by sex, age, race and ethnicity, and body mass index: United States, 2003-2006. Division of Health and Nutriition Examination Surveys. Natl Health Stat Report, (13):1-7.

Expert Panel on Detection, Evaluation and Treatment of High Blood Cholesterol in Adults (2001). Executive summary of the Third Report of the National Cholesterol Education Program (NCEP) Expert Panel on Detection, Evaluation and Treatment of High Cholesterol. JAMA, 285:2486-97. 
Ferreira, S.R.G., Gimeno, S.G.A., Hirai, A.T., Harima, H., Matsumura, L., \& Pittito, B.A. (2008). Effects of an intervention in eating habits and physical activity in Japanese-Brazilian women with a high prevalence of metabolic syndrome in Bauru, São Paulo State, Brazil. Cad. Saúde Pública, 24(Sup 2):294302.

Folstein, M.F., Folstein, S.E., \& Mchugh, P.R. (1975). "Mini-mental state" A practical method for grading the cognitive state of patients for the clinician. Journal of Psychiatric Research, 12(3):189-198.

Fox, C.S., Massaro, J.M., Hoffmann, U., Pou, K.M., Horvat-Maurovich, P., Liu, C.Y. et al. (2007). Abdominal visceral and subcutaneous adipose tissue compartments: association with metabolic risk factors in the Framingham Heart Study. Circulation, 116(1):39-48.

Franco, G.P.P., Scala, L.C.N., Alves, C.J., França, V.G.A., Cassanelli, T., \& Jardim, P.C.B.V. (2009). Síndrome metabólica em Hipertensos de Cuiabá - MT: Prevalência e Fatores Associados. Arq Brasil Cardiol, 92(6):472-8.

Góis, A.L.B. \& Veras, R.P. (2010). Informações sobre a morbidade hospitalar em idosos nas internações do Sistema Único de Saúde do Brasil. Ciên Saúde Colet, 15(6): 2859-69.

Gragnolati, M., Jorgensen, O.H., Rocha, R., \& Fruttero, A. (2011). Growing old in an older Brazil: implications of population aging on growth, poverty, public finance and service delivery. Washington: The World Bank.

Gronner, M.F., Bosi, P.L., Carvalho, A.M., Casale, G., Contrera, D., Pereira, M.A., Diogo, T.M., Torquato, M.T.C.G., Souza, G.M.D., Oishi, J., \& Leal, A.M.O. (2011). Prevalence of metabolic syndrome and its association with educational inequalities among Brazilian adults: a population-based study. Braz $J$ Med Biol Res, 4:713-9.

Janssen, I., Powell, L.H., Kazlauskaite, R. \& Dugan, S.A (2010). Testosterone and visceral fat in midlife women: the Study of Women's Health Across the Nation (SWAN) fat patterning study. Obesity (Silver Spring), 18:604-10.

Lebrão, M.L., \& Duarte, Y.A.O. (2003). SABE - Saúde, Bem-estar e Envelhecimento - O Projeto Sabe no município de São Paulo: uma abordagem inicial. Brasília: Organização Pan-Americana de Saúde.

Leitão, M.P.C., \& Martins, I.S. (2012). Prevalência e fatores associados à Síndrome Metabólica em usuários de Unidades Básicas de Saúde em São Paulo. Rev Assoc Med Bras, 58(1):60-9.

Lima, C.R.O.C., Araújo, L.S., Lemaire, D.C., Rios, DL, Conceição, G.C.; Brandão, N.A., \& Araújo, E.M.Q. (2017). Associação entre níveis séricos de vitamina $\mathrm{D}$ e componentes da síndrome metabólica em pacientes atendidos no centro de estudos e atendimento dietoterápico da Universidade do Estado da Bahia. Ciências Médicas e Biológicas, 16(3):367-373.

Malachias, M.V., Souza, W.K., Plavnik, F.L., et al. (2016). Sociedade Brasileira de Cardiologia. $7^{\text {a }}$ Diretriz brasileira de hipertensão arterial. Arq. Bras. Cardiol., 107(3 supl 3):1-83.

Mazo, G.Z., \& Benedetti, T.R.B. (2010). Adaptação do questionário internacional de atividade física para idosos. Bras Cineantropom Desempenho Hum., 12(6):480-4.

Mendes, K.G., Theodoro, H., Rodrigues, A.D., \& Olinto, M.T.A. (2012). Prevalência de síndrome metabólica e seus componentes na transição menopáusica: uma revisão sistemática. Cad. Saúde Pública, 28(8):1423-37.

Nichols, G.A., \& Moler, E.J. (2011). Metabolic syndrome components are associated with future medical costs independent of cardiovascular hospitalization and incident diabetes. Metab Syndr Relat Disord, 9(2):127-33.

Paula, H.A.A., Ribeiro, R.C.L., Rosado, L.E.F.P.L., Pereira, R.S.F., \& Franceschini, S.C.C. (2010). Comparação de Diferentes Critérios de Definição para Diagnóstico de Síndrome Metabólica em Idosas. Arq Bras Cardiol, 95(3):346-53.

Paula, J.A.T., Moreira, O.C., Silva, C.D., Silva, D.S., \& Amorim, P.R.S. (2015). Metabolic syndrome prevalence in elderly of urban and rural communities participants in the HIPERDIA in the city of Coimbra/MG, Brazil. Invest Educ Enferm, 33(2):326-32.

Pimenta, A.M., Gazzinelli, A., \& Velásquez-Melendez, G. (2011). Prevalência da síndrome metabólica e seus fatores associados em área rural de Minas Gerais (MG, Brasil). Ciênc saúde colet, 16(7):3297-306.

Pradhan, A.D. (2014). Sex differences in the metabolic syndrome: implications for cardiovascular health in women. Clin Chem, 60(1):44-52.

Rigo, J.C., Vieira, J.L., Dalacorte, R.R., \& Reichert, C.L. (2009). Prevalência de Síndrome Metabólica em Idosos de uma Comunidade: Comparação entre três métodos diagnósticos. Arq Bras Cardiol, 93(2):85-91.

Rosa, C.B., Agostini, J.Á., Bianchi, P.D., Garces, S.B.B., Hansen, D., Moreira, P.R., \& Schwanke, C.H.A. (2016). Síndrome metabólica e estado nutricional de idosos cadastrados no HiperDia. Sci Med, 26(3):ID23100.

Saad, M.A.N., Cardoso, G.P., Martins, W.A., Velarde, L.G.C., \& Cruz Filho, R.A. (2014). Prevalence of metabolic syndrome in elderly and agreement among four diagnostic criteria. Arq Bras Cardiol, 102(3)263-9.

Saukkonen, T., Jokelainen, J., Timonen, M., Cederberg, H., Laakso, M., Harkonen, P., Keinänen-Kiukaanniemi, S., \& Rajala, U. (2012). Prevalence of metabolic syndrome components among the elderly using three different definitions: a cohort study in Finland. Scand J Prim Health Care, 30(1):29-34.

Silva, C.G., Rodrigues, J.B., Medeiros Júnior, J.R., Coutinho, N.P.S., Lopes, M.L.H., \& Sardinha, A.H.L. (2012). Perfil dos idosos com síndrome metabólica. Rev Pesq Saúde, 13(2):17-20.

Souza, T. S., Nepomuceno, A. F. S. F., Costa, S. M., Oliveira, Y. N. S., Carneiro, J. A. O., \& Casotti, C. A. (2021). Potential drug interactions in the elderly with metabolic syndrome. Research, Society and Development, 10(9), e29410918080. 
Research, Society and Development, v. 10, n. 13, e189101319190, 2021

(CC BY 4.0) | ISSN 2525-3409 | DOI: http://dx.doi.org/10.33448/rsd-v10i13.19190

Tavares, D.S., Gomes, N.C., Rodriguês, L.R., \& Tavares, D.M.S. (2018). Perfil de idosos com síndrome metabólica e fatores associados às possíveis interações medicamentosas. Rev Brasileira de Geriatria e Gerontologia, 21(2):164-175.

Veras, R., \& Dutra, S. (2008). Perfil do idoso brasileiro: questionário BOAS. Rio de Janeiro; UERJ. UnATI. 98p.

Veras, R.P. \& Oliveira, M. (2018). Aging in Brazil: the building of a healthcare model. Rev Ciência \& Saúde Coletiva, 23(6):1929-36.

Vieira, E.C., Peixoto, M.R.G., \& Silveira, E.A. (2014). Prevalência e fatores associados à Síndrome Metabólica em idosos usuários do Sistema Único de Saúde. Rev bras epidemiol, 17(4):805-17.

Wachholz, P.A., \& Masuda, P.Y. (2009). Caracterização e prevalência de síndrome metabólica em idosos, segundo dois critérios diagnósticos diferentes. Estud interdiscipl envelhec, 14(1):95-106. 